\title{
Myeloablative Conditioning with PBSC Grafts for T Cell-Replete Haploidentical Donor Transplantation Using Posttransplant Cyclophosphamide
}

\author{
Scott R. Solomon, Melhem Solh, Lawrence E. Morris, H. Kent Holland, and Asad Bashey \\ Blood and Marrow Transplant Program at Northside Hospital, Atlanta, GA 30342, USA \\ Correspondence should be addressed to Scott R. Solomon; ssolomon@bmtga.com
}

Received 3 November 2015; Accepted 29 December 2015

Academic Editor: Franco Aversa

Copyright (C) 2016 Scott R. Solomon et al. This is an open access article distributed under the Creative Commons Attribution License, which permits unrestricted use, distribution, and reproduction in any medium, provided the original work is properly cited.

\begin{abstract}
Relapse is the main cause of treatment failure after nonmyeloablative haploidentical transplant (haplo-HSCT). In an attempt to reduce relapse, we have developed a myeloablative (MA) haplo-HSCT approach utilizing posttransplant cyclophosphamide (PT/Cy) and peripheral blood stem cells as the stem cell source. We summarize the results of two consecutive clinical trials, using a busulfan-based $(n=20)$ and a TBI-based MA preparative regimen $(n=30)$, and analyze a larger cohort of 64 patients receiving MA haplo-HSCT. All patients have engrafted with full donor chimerism and no late graft failures. Grade III-IV acute GVHD and moderate-severe chronic GVHD occurred in 23\% and 30\%, respectively. One-year NRM was 10\%. Predicted three-year overall survival, disease-free survival, and relapse were 53\%, 53\%, and 26\%, respectively, in all patients and 79\%, $74 \%$, and $9 \%$, respectively, in patients with a low/intermediate disease risk index (DRI). In multivariate analysis, DRI was the most significant predictor of survival and relapse. Use of TBI (versus busulfan) had no significant impact on survival but was associated with significantly less BK virus-associated hemorrhagic cystitis. We contrast our results with other published reports of MA haplo-HSCT PT/Cy in the literature and attempt to define the comparative utility of MA haplo-HSCT to other methods of transplantation.
\end{abstract}

\section{Introduction}

Seventy percent of patients who urgently need an allogeneic hematopoietic stem cell transplantation (HSCT) do not have an available HLA-matched sibling donor. In such patients, a search for an HLA-matched unrelated donor (MUD) can identify an 8/8 HLA-identical donor for approximately $30 \%$ to $40 \%$ of transplant recipients. The probability of finding an acceptable MUD varies by ethnic groups, ranging from $75 \%$ in the white Europeans, to $30 \%$ to $40 \%$ in the Mexican and Central/South Americans, to $15 \%$ to $20 \%$ for the African Americans and black Caribbeans [1]. In addition, MUD transplantation is also complicated by the amount of time it takes from search initiation to transplantation, causing some patients to relapse or physically deteriorate while waiting for transplantation. In contrast, a haploidentical family member (haplo) can be identified and rapidly utilized in nearly all cases.
Historically, HSCT from a partially HLA-mismatched relative has been complicated by unacceptably high incidences of graft rejection, severe graft-versus-host disease (GVHD), and nonrelapse mortality (NRM) $[2,3]$. To address the risk of graft rejection and GVHD, extensive T cell depletion has been utilized in association with antithymocyte globulin (ATG) and high peripheral blood stem cell (PBSC) dose [4]; however, NRM from infectious complications remains a challenge. More recently, the investigators at Johns Hopkins University have pioneered a method to selectively deplete alloreactive cells in vivo by administering high doses of cyclophosphamide (Cy) in a narrow window after transplantation [5]. After nonmyeloablative (NMA) conditioning, this approach has resulted in low NRM (4\% and 15\% at 1 and 2 years, resp.), because of low rates of GVHD and infectious complications. Immune reconstitution was promising with low risk of cytomegalovirus (CMV) or invasive mold infections. Using high-dose, posttransplantation cyclophosphamide (PT/Cy), 
crossing the HLA barrier in HSCT is now feasible without the need for extensive $\mathrm{T}$ cell depletion or serotherapy.

Studies of NMA haplo-HSCT with PT/Cy show remarkable tolerability of this approach with low rates of GVHD, infection, and NRM. Relapse of malignancy remains the predominant cause of treatment failure, occurring in approximately $45 \%$ to $51 \%$ of patients $[5,6]$. NMA haplo-HSCT with $\mathrm{PT} / \mathrm{Cy}$ has also been associated with an approximately $10 \%$ rate of engraftment failure resulting in autologous recovery. The use of more intense/myeloablative (MA) preparative regimens and $\mathrm{PBSC}$ grafts may potentially reduce the rate of relapse and graft rejection following haplo-HSCT PT/Cy transplants. However, only a limited number of such studies have been reported. In this paper, we report our experience with MA conditioning and PBSC allografts for T-replete haplo-HSCT using PT/Cy. We define the major predictors of outcome following this strategy. We also describe other published reports of MA haplo-HSCT PT/Cy in the literature. Finally, we compare the outcomes of MA and NMA haploHSCT using PT/Cy and attempt to define the comparative utility of MA haplo-HSCT in relation to MUD transplantation.

\section{Busulfan-Based MA Haplo-HSCT (NSH 864 Protocol)}

In a proof-of-principle study of MA haplo-HSCT, twenty patients with high risk hematologic malignancies were treated with a preparative regimen of fludarabine $\left(125-180 \mathrm{mg} / \mathrm{m}^{2}\right)$, i.v. busulfan $\left(440-520 \mathrm{mg} / \mathrm{m}^{2}\right)$ and Cy $(29 \mathrm{mg} / \mathrm{kg})$ before transplant, a G-CSF-mobilized PBSC graft, and posttransplant GVHD prophylaxis comprised of Cy $50 \mathrm{mg} / \mathrm{kg} / \mathrm{d}$ on $\mathrm{d}$ +3 and +4 , MMF $15 \mathrm{mg} / \mathrm{kg}$ three times daily $\mathrm{d}+5-+35$, and tacrolimus (target $5-15 \mathrm{ng} / \mathrm{mL}$ ) days +5 to +180 [7]. The median age of patients was 44 years (range: $25-56$ years). Eleven patients (55\%) underwent HSCT with relapsed/refractory disease (acute myelogenous leukemia [AML] 5, chronic myelogenous leukemia-blast crisis [CML-BC] 1, acute lymphoblastic leukemia 2, non-Hodgkin lymphoma 1, Hodgkin's disease 1 , and chronic lymphocytic leukemia/Richters 1 ). The remaining patients had either AML CR1 with poor-risk cytogenetics and/or induction failure or chronic myelogenous leukemia resistant to all tyrosine kinase inhibitors.

All patients engrafted and demonstrated 100\% donor chimerism in both peripheral blood $\mathrm{T}$ cell and myeloid cells from day +30 . Cumulative incidence of one-year NRM was $10 \%$ and that of grade III-IV acute GVHD and severe chronic GVHD was $10 \%$ and $5 \%$, respectively. Relapse was acceptable, occurring in $40 \%$ of patients, despite the fact that the majority had relapsed/refractory disease at time of transplant. With a median follow-up of 20 months, estimated probabilities of overall and disease-free survival (DFS) were $69 \%$ and $50 \%$, respectively.

There were no cases of invasive mold infections or EBVrelated PTLD. Only one patient had CMV disease and only one patient died of a viral infection (parainfluenza 3) suggesting that anti-infection immunity was preserved with this approach. However, nonfatal BK virus-associated hemorrhagic cystitis (HC) was seen in $75 \%$ of patients at a median of

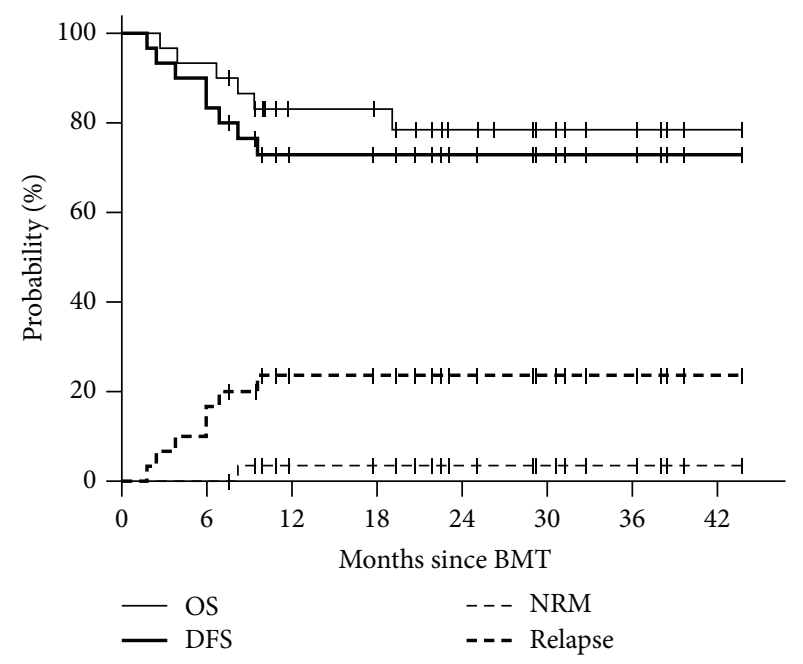

Figure 1: Kaplan-Meier analysis of overall survival, disease-free survival, and nonrelapse mortality and following TBI-based MA haplo-HSCT.

38 days after transplant. Although it is not a life-threatening complication, it was a source of significant morbidity for some patients. We hypothesized that $\mathrm{HC}$ was predisposed to by the combined effect of high-dose busulfan and PT/Cy.

\section{Total-Body Irradiation-Based Haplo-HSCT (NSH 922 Protocol)}

In an attempt to reduce the risk of BK virus-associated $\mathrm{HC}$, thirty patients were enrolled on prospective phase II trial utilizing a TBI-based myeloablative preparative regimen (fludarabine $25 \mathrm{mg} / \mathrm{m}^{2} / \mathrm{d} \times 3 \mathrm{~d}$ and TBI $150 \mathrm{cGy}$ bid on $\mathrm{d}-4$ to -1 [total dose $1200 \mathrm{cGy}$ ]) followed by infusion of unmanipulated peripheral blood stem cells from a haploidentical family donor [8]. Postgrafting immunosuppression again consisted of Cy $50 \mathrm{mg} / \mathrm{kg} /$ day on days 3 and 4, MMF through d 35, and tacrolimus through d 180. Median patient age was 46.5 years (range 24-60). Transplant diagnosis included AML [9], ALL [6], CML [5], MDS [1], and NHL [2]. Using the revised Dana-Farber/CIBMTR disease risk index (DRI), patients were classified as having low [4], intermediate [10], high [11], and very high [3] risk.

All patients engrafted with a median time to neutrophil and platelet recovery of 16 and 25 days, respectively. All evaluable patients achieved sustained complete donor $\mathrm{T}$ cell and myeloid chimerism by day +30 . Acute GVHD, grades IIIV and III-IV, was seen in $43 \%$ and $23 \%$, respectively. The cumulative incidence of moderate-to-severe chronic GVHD was $22 \%$ (severe in 10\%). Nonrelapse mortality (NRM) at 2 years was $3 \%$, which consisted of one death due to noninfectious respiratory failure/ARDS 8 months after transplant in a patient with chronic GVHD. Estimated two-year survival, DFS, and relapse were $78 \%, 73 \%$, and $24 \%$, respectively (Figure 1). Two-year DFS and relapse rate in patients with low/intermediate disease risk, determined by the DRI, were 


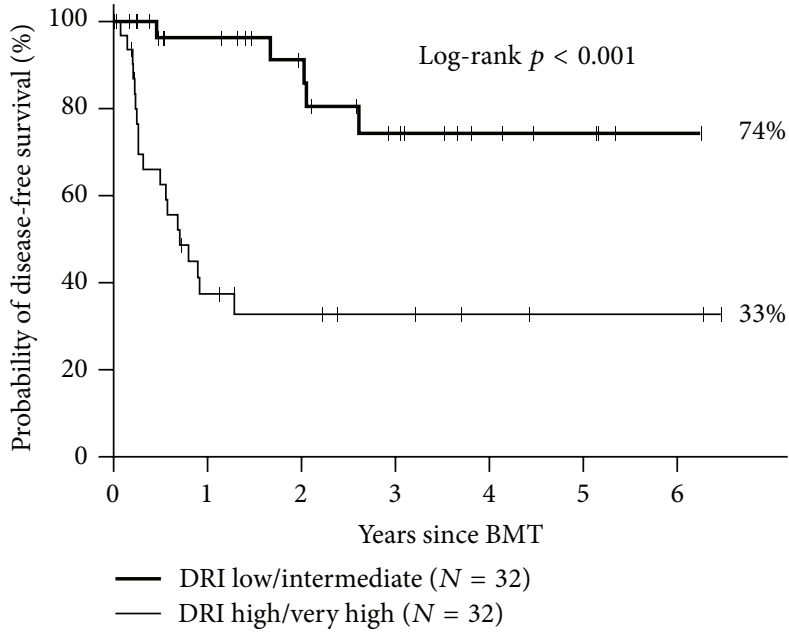

(a)

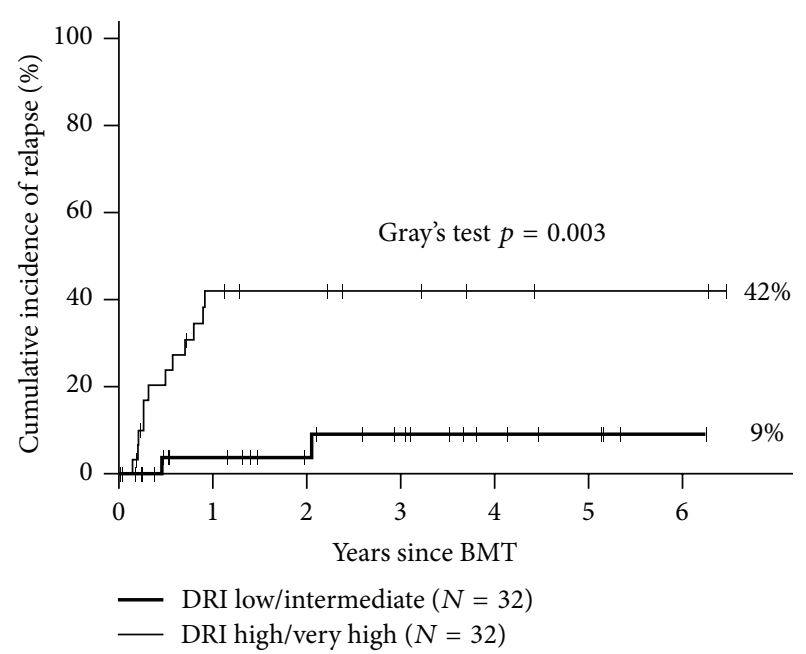

(b)

FIGURE 2: Effect of disease risk index on (a) disease-free survival and (b) relapse following MA haplo-HSCT.

$100 \%$ and $0 \%$, respectively, compared with $39 \%$ and $53 \%$ for patients with high/very high risk disease.

As noted in our prior experience with busulfan-based MA haplo-HSCT, posttransplant fever was common and occurred in the first 5 posttransplant days in nearly all patients. Fevers resolved in all patients following administration of PT/Cy. CMV reactivation ( $\geq 400$ copies/mL) occurred in $15 / 26(58 \%)$ of at-risk patients (either donor or recipient with CMV positive serostatus) at a median of day +43 after transplant (range 11-157). CMV disease did not occur. There were no episodes of invasive mold infection or infectious death in the first 100 days after transplant. There were no cases of EBV reactivation. BK virus-associated HC of any grade occurred in 30\% of patients and was severe (grade $\geq 3$ ) in $7 \%$. As compared with our previous experience with busulfan-based MA haploHSCT, HC occurred significantly less often following TBIbased MA haplo-HSCT (any grade: $30 \%$ versus $75 \%, p=$ 0.005 ; severe HC: $7 \%$ versus $30 \%, p=0.037)$.

\section{Predictors of Outcome following MA Haplo-HSCT and PT/Cy}

In order to determine predictors of outcome following MA haplo-HSCT and PT/Cy, we evaluated that sixty-four consecutive patients have been transplanted following either busulfan-based ( $n=20$; NSH 864 ) or TBI-based ( $n=44$; including 30 patients on NSH 922 and the remaining 14 patients treated identically after completion of the trial) MA conditioning, T cell-replete PBSC infusion, PT/Cy, and tacrolimus/mycophenolate mofetil. Median age of the cohort was 43 years (range 21-60). Patient characteristics included a high/very high disease risk by the Dana-Farber/CIBMTR disease risk index (DRI) in 32 patients (50\%), KPS $<90$ in 69\%, and comorbidity index (CMI) of $\geq 2$ in $58 \%$ of patients. The most common indications for transplant were AML, ALL, and advanced-phase CML in 55\%, 20\%, and $12 \%$ of patients, respectively. Median follow-up for surviving patients was 24 months.

All patients engrafted with full donor chimerism and no late graft failures. Grade II-IV, III-IV acute GVHD and moderate-severe chronic GVHD occurred in 46\%, 23\%, and $30 \%$, respectively. One-year NRM was $10 \%$. Predicted threeyear overall survival (OS), disease-free survival (DFS), and relapse are $53 \%, 53 \%$, and $26 \%$, respectively. In the 32 patients with standard risk disease (low/intermediate DRI), outcomes were significantly improved with one-year NRM of $0 \%$ and predicted 3-year OS, DFS, and relapse of 79\%, 74\%, and $9 \%$, respectively (Figure 2 ).

In multivariate analysis, high/very high DRI was the most significant negative predictor of OS (HR 13.26, $p<0.001$ ), followed by $\mathrm{CMI} \geq 2(\mathrm{HR} 3.54, p=0.01)$ and age $(\mathrm{HR}$ $1.26, p=0.038$, per 5-year increase in age). DRI was also significantly associated with DFS (HR 10.84, $p<0.001$ ), NRM (HR 15.0, $p=0.004)$, and relapse (HR 8.85, $p=0.004)$ (Table 1). Conditioning regimen (TBI versus busulfan) had no significant impact on OS, DFS, NRM, or relapse.

\section{Additional Published Experience with MA Haplo-HSCT and PT/Cy}

Several other groups have published similar experiences with MA haplo-HSCT with PT/Cy. Grosso et al. [12] reported a "two-step" strategy where a defined dose of haploidentical T cells $(2 \times 108 / \mathrm{kg})$ was infused after MA doses of TBI. Patients then received $60 \mathrm{mg} / \mathrm{kg}$ of $\mathrm{CY}$ on two consecutive days, followed later by infusion of highly purified CD34+ cells from the donor. All patients engrafted and the cumulative incidence of grade III-IV acute GVHD and NRM was 7.4\% and $22.5 \%$, respectively, for the 27 patients treated. With a median follow-up of 40 months, overall survival was $48 \%$. A second study from the same group [13], which included only patients in remission at the time of transplant, demonstrated 
TABLE 1: Predictors of transplant outcomes following MA haplo HSCT.

\begin{tabular}{|c|c|c|c|c|c|c|c|c|}
\hline & \multicolumn{2}{|c|}{ OS } & \multicolumn{2}{|c|}{ DFS } & \multicolumn{2}{|c|}{ NRM } & \multicolumn{2}{|c|}{ Relapse } \\
\hline & HR & $p$ & HR & $p$ & $\mathrm{HR}$ & $p$ & HR & $p$ \\
\hline DRI (high versus low/int) & 13.26 & $<0.001$ & 10.84 & $<0.001$ & 15.0 & 0.004 & 8.85 & 0.004 \\
\hline CMI ( $\geq 2$ versus $<2$ ) & 3.54 & 0.010 & 3.09 & 0.018 & 13.6 & 0.007 & - & - \\
\hline Age $(<50$ versus $\geq 50)$ & 1.26 & 0.038 & 1.31 & 0.015 & 1.43 & 0.055 & - & - \\
\hline
\end{tabular}

The following variables were considered in Cox analysis: age, diagnosis, Karnofsky performance status (KPS), comorbidity index (CMI), revised Dana-Farber disease risk index (DRI), conditioning regimen (busulfan versus TBI), year of transplant, acute GVHD, and chronic GVHD. Variables were selected by $10 \%$ threshold. Acute and chronic GVHD were modeled as time-dependent variables.

a 2 yr NRM, relapse, and PFS of $4 \%, 19 \%$, and $74 \%$, respectively. The requirement for stringent ex vivo $\mathrm{T}$ depletion of the hematopoietic cell product differentiates this approach and may limit its widespread applicability. Furthermore, given the resistance of hematopoietic stem cells to $\mathrm{Cy}$, such delayed infusion of selected CD34+ cells may be unnecessary.

Symons et al. [11] reported on 97 patients with either leukemias in complete remission or lymphoma with chemosensitive disease. Patients received MA haplo-HSCT PT/Cy utilizing bone marrow grafts. The preparative regimen consisted of IV busulfan (pharmacokinetically adjusted) on days -6 to -3 and Cy $(50 \mathrm{mg} / \mathrm{kg} /$ day) on days -2 and -1 , except for patients with acute lymphocytic leukemia or lymphoblastic lymphoma who received Cy $(50 \mathrm{mg} / \mathrm{kg} /$ day $)$ on days -5 and -4 and TBI (200 cGy twice daily) on days -3 to -1 . Donor engraftment occurred in 73/82 (89\%) patients. Estimated probabilities of NRM and grade III-IV acute GVHD at 100 days were $11 \%$ and $7 \%$, respectively. The cumulative incidence of relapse was $44 \%$. With a median follow-up of surviving patients of 474 days, estimated $2 \mathrm{yr}$ overall and disease-free survival is $57 \%$ and $49 \%$, respectively.

Raiola et al. [10] reported on 50 patients receiving a MA haplo-HSCT PT/Cy utilizing bone marrow grafts. The regimens used were thiotepa, busulfan, and fludarabine $(n=$ $35)$ or TBI and fludarabine $(n=15)$. Forty-five patients $(90 \%)$ engrafted with an 18-month cumulative incidence of NRM, relapse, and PFS of $18 \%, 22 \%$, and 51\%, respectively. PFS was $67 \%$ for patients transplanted in remission versus $37 \%$ for patients with active disease. Reported incidences of acute and chronic GVHD were low. As in our experience, HC was more common in patients receiving busulfan rather than TBIbased conditioning.

Whether PBSC or BM is the preferred stem cell source following MA haplo-HSCT remains unclear; however BM appears to be associated with a higher rate of graft failure, occurring in approximately $10 \%$ of patients in both the series by Raiola et al. [10] and the experience of Symons et al. [11]. Graft failure has not been reported with PBSC based myeloablative haplo-HSCT and PT/Cy.

\section{Comparison of MA and NMA Haplo-PT/Cy}

The overall risk of relapse associated with MA haplo-HSCT in the majority of studies is $20-25 \%$ [7, 8, 10, 13] and compares favorably with that reported for NMA haplo-HSCT (45$51 \%)[5,6]$. In our analysis of 64 patients receiving MA haplo-HSCT, relapse risk in patients with low $(n=7)$ or intermediate ( $n=25)$ DRI was $9 \%$, compared with $42 \%$ relapse rate in high $(n=24)$ or very high $(n=8)$ DRI patients. This compares favorably to that seen in the NMA setting, where relapse risk according to DRI was recently analyzed in 372 consecutive patients by the group from Johns Hopkins University [14]. In this analysis, the risk of relapse was also highly correlated with DRI, with relapse occurring in approximately $75 \%, 50 \%$, and $20 \%$ of patients in the high/ very high, intermediate, and low DRI groups, respectively. The finding of higher relapse following NMA conditioning parallels what has been seen following matched related or unrelated donor transplantation [9, 15-17].

\section{Comparison of MA Haplo-PT/Cy with MA MUD Transplants}

In order to evaluate the comparative efficacy of MA haploHSCT, we have compared outcomes of patients receiving TBI-based MA haplo-HSCT with PT/Cy $(n=30)$ with a contemporaneously treated cohort of consecutive patients at our institution receiving HLA-matched (8/8 HLA-A, HLA-B, HLA-C, and HLA-DR) MA T cell-replete MUD transplantation $(n=48)$ [8]. Haplo- and MUD transplant patients were well matched according to age, diagnosis, disease risk, CMV serostatus, and comorbidity index. The groups did differ in the use of PBSC as the stem cell source which was utilized in all haplotransplant recipients compared with 32 of 48 MUD transplants recipients. When compared with recipients of MA MUD transplants, outcomes after MA haplo-HSCT were statistically similar to $2 \mathrm{yr}$ OS and DFS being $78 \%$ and $73 \%$, respectively, after haplotransplant versus $71 \%$ and $64 \%$, respectively, after MUD transplants. Grade II-IV acute GVHD was seen less often following haplotransplantation compared with MUD transplantation (43\% versus 63\%, $p=$ $0.049)$, as was moderate-to-severe chronic GVHD $(22 \%$ versus $58 \%, p=0.003)$. The lower incidence of chronic GVHD occurred despite the greater use of PBSC in the haploHSCT group.

Similarly, a Center for International Blood and Marrow Transplant Research (CIBMTR) analysis [18] compared outcomes of adults with acute myeloid leukemia (AML) after haplo- $(n=192)$ and MUD $(n=1982)$ transplantation, including 104 MA haplotransplants and 1245 MA MUD transplants. In this large analysis, there were no significant differences in 1 yr NRM (12\% versus $14 \%)$, 3 yr relapse $(44 \%$ versus $39 \%$ ), or 3 yr OS (46\% versus $44 \%$ ), comparing MA haplo- and MA MUD transplants, respectively. Grade II-IV 
acute GVHD (16\% versus 33\%), grade III-IV acute GVHD (7\% versus 13\%), and chronic GVHD (30\% versus 53\%) were all statistically lower in haplopatients compared with MUD patients.

\section{Immune Recovery following MA Haplo-PT/Cy}

Historically, MA haplotransplantation has been associated with considerable infectious morbidity and mortality. In contrast, our experience and others suggest that MA haplo$\mathrm{PT} / \mathrm{Cy}$ may significantly reduce the risk of infectious complications. In a published series of thirty patients undergoing TBI-based MA haplo-PT/Cy [8], CMV reactivation ( $\geq 400$ copies/mL) occurred in only 15/26 (58\%) of at-risk patients (either donor or recipient with CMV positive serostatus), and CMV disease did not occur. There were no episodes of invasive mold infection or infectious death in the first 100 days after transplant. Furthermore, there were no cases of EBV, HHV6, or adenovirus infections.

The reduced risk of infectious complications following MA haplo-PT/Cy has translated into low NRM, approximately $10 \%$ in the first year after transplant. Our experience compares favorably to the results reported with T cell-depleted (TCD) MA haplo, where NRM of approximately $40 \%$ have been seen, with much of this attributable to infectious mortality [4, 19-21]. Ciurea and colleagues at the MD Anderson Cancer Center analyzed their outcomes following MA haplo-PT/Cy following a preparative regimen of fludarabine, melphalan, and thiotepa, with historical results of TCD MA haplo using the same preparative regimen [20]. In this analysis, one-year NRM favored PT/Cy (16\% versus $42 \%)$ as did death directly attributable to infection (9\% versus $24 \%$ ), with significantly less viral and fungal infections seen in $\mathrm{PT} / \mathrm{Cy}$ versus TCD patients. T cell subset analysis demonstrated significant improvements in $\mathrm{T}$ cell recovery in $\mathrm{PT} / \mathrm{Cy}$ versus TCD patients, with more rapid reconstitution noted in multiple T cell subsets (CD4, CD8, naïve, and memory).

Immune reconstitution following haplo-PT/Cy is characterized by a diverse $\mathrm{T}$ cell receptor repertoire and appears dependent on $\mathrm{T}$ memory stem cells maturing from naïve $\mathrm{T}$ cells $[22,23]$. These cells are adoptively transferred in the donor graft and have been shown to survive cyclophosphamide-induced deletion. Furthermore, regulatory $\mathrm{T}$ cells also are preferentially preserved following PT/Cy, likely due to higher aldehyde dehydrogenase in these cells [24]. Finally, murine studies have demonstrated that PT/Cy relatively spares pathogen and cancer-specific T cells [25]. The selective elimination of alloreactive donor $T$ cells with relative preservation of nonalloreactive donor $\mathrm{T}$ cell clones provides a mechanistic understanding of the surprisingly low infectious mortality following MA haplo-PT/Cy.

\section{Discussion}

In the past decade, there has been a growing interest in the use of haplo-HSCT due to the rapid and nearly universal availability of donors, which is a critical issue in patients with advanced hematologic malignancies. A major advance in the success of haplo-HSCT is the use of properly timed PT/Cy, a technique pioneered by investigators at Johns Hopkins University $[5,26]$. Using a NMA approach, this strategy has resulted in low rates of GVHD, infection, and NRM. However, relapse remains the major cause of treatment failure, occurring in approximately half of transplant recipients. One explanation for the high rate of relapse, as in other NMA HSCT trials, is that the transplantation conditioning was not intense enough to achieve sufficient tumor cytoreduction.

In order to reduce the risk of relapse in patients with high risk hematologic malignancies, our group and others have demonstrated the feasibility of performing MA haplo-HSCT utilizing PT/Cy. In 64 consecutive patients transplanted at our institution following either busulfan-based $(n=20)$ or TBI-based ( $n=44$ ) MA conditioning, we have noted universal engraftment with rapid donor chimerism, acceptable rates of GVHD (grade III-IV acute GVHD and moderatesevere chronic GVHD occurred in $23 \%$ and $30 \%$, resp.), and a low one-year NRM of $10 \%$. Predicted three-year overall survival (OS), disease-free survival (DFS), and relapse were $53 \%, 53 \%$, and $26 \%$, respectively, and in the 32 patients with standard risk disease (low/intermediate DRI), outcomes were very favorable (3-year OS, DFS, and relapse of 79\%, 74\%, and 9\%, resp.).

Relapse appears less following MA conditioning with relapse rates in the majority of studies of $20-25 \%[7,8,10,13]$, compared with that reported for NMA haplo-BMT (45-51\%) $[5,6]$. However, truly defining the influence of the preparative regimen intensity on relapse risk will likely require a randomized controlled trial. When comparing our results with the other published experiences of MA haplo-HSCT using PT/Cy, it becomes evident that disease risk, as defined by either the DRI or disease status at the time of transplant, is the primary driver of outcomes, with $2 \mathrm{yr}$ DFS being approximately $67-74 \%[8,10,13]$ in patients transplanted in remission without high risk disease defined by the DRI. Whether PBSC or BM is the preferred stem cell source following myeloablative haplo-HSCT remains unclear; however BM appears to be associated with a higher rate of graft failure, occurring in approximately $10 \%$ of patients $[10,11]$ receiving marrow grafts, and is obviously more consequential following MA conditioning.

Although there have been no randomized studies to date, there is now compelling evidence regarding the equivalent efficacy and safety of haplo-HSCT PT/Cy and MUD transplantation, in both the NMA and MA setting [8, 18, 27-29]. When considering the optimal transplant donor type, MUD versus haplo-HSCT, one must consider the inherent advantages of haplodonors including near universal and rapid availability, as well as lower costs related to donor searching and graft acquisition, whereas as almost all patients have an available haplomatched family member, the availability of an 8/8 matched unrelated donor varies according to ethnic background, ranging from $75 \%$ for white patients of European descent to less than $20 \%$ for the African Americans. Furthermore, given the complexities inherent in registry searching, time from initiation of donor searching to transplant can be significant, averaging around 3 months. 
In conclusion, our results show that MA haplo-HSCT results in favorable engraftment, acceptable rates of GVHD, and low nonrelapse mortality. Relapse rates appear lower than that reported with NMA haplo-HSCT. DRI represents the strongest predictor of outcome following MA haplo-HSCT and PT/Cy. Disease-free and overall survival is equivalent to recipients of MA MUD transplants. Therefore, in younger patients without contraindications to standard intensity conditioning, MA haplo-HSCT is a valid option for patients with advanced hematologic malignancies who lack timely access to a conventional donor.

\section{Conflict of Interests}

The authors declare that there is no conflict of interests regarding the publication of this paper.

\section{References}

[1] L. Gragert, M. Eapen, E. Williams et al., "HLA match likelihoods for hematopoietic stem-cell grafts in the U.S. registry," The New England Journal of Medicine, vol. 371, no. 4, pp. 339348, 2014

[2] P. G. Beatty, R. A. Clift, E. M. Mickelson et al., "Marrow transplantation from related donors other than HLA-identical siblings," The New England Journal of Medicine, vol. 313, no. 13, pp. 765-771, 1985.

[3] R. Szydlo, J. M. Goldman, J. P. Klein et al., "Results of allogeneic bone marrow transplants for leukemia using donors other than HLA-identical siblings," Journal of Clinical Oncology, vol. 15, no. 5, pp. 1767-1777, 1997.

[4] F. Aversa, A. Terenzi, A. Tabilio et al., "Full haplotype-mismatched hematopoietic stem-cell transplantation: a phase II study in patients with acute leukemia at high risk of relapse," Journal of Clinical Oncology, vol. 23, no. 15, pp. 3447-3454, 2005.

[5] L. Luznik, P. V. O’Donnell, H. J. Symons et al., "HLAhaploidentical bone marrow transplantation for hematologic malignancies using nonmyeloablative conditioning and highdose, posttransplantation cyclophosphamide," Biology of Blood and Marrow Transplantation, vol. 14, no. 6, pp. 641-650, 2008.

[6] C. G. Brunstein, E. J. Fuchs, S. L. Carter et al., "Alternative donor transplantation after reduced intensity conditioning: results of parallel phase 2 trials using partially HLA-mismatched related bone marrow or unrelated double umbilical cord blood grafts," Blood, vol. 118, no. 2, pp. 282-288, 2011.

[7] S. R. Solomon, C. A. Sizemore, M. Sanacore et al., "Haploidentical transplantation using $\mathrm{T}$ cell replete peripheral blood stem cells and myeloablative conditioning in patients with high-risk hematologic malignancies who lack conventional donors is well tolerated and produces excellent relapse-free survival: results of a prospective phase II trial," Biology of Blood and Marrow Transplantation, vol. 18, no. 12, pp. 1859-1866, 2012.

[8] S. R. Solomon, C. A. Sizemore, M. Sanacore et al., "Total body irradiation-based myeloablative haploidentical stem cell transplantation is a safe and effective alternative to unrelated donor transplantation in patients without matched sibling donors," Biology of Blood and Marrow Transplantation, vol. 21, no. 7, pp. 1299-1307, 2015.

[9] O. Ringdén, M. Labopin, G. Ehninger et al., "Reduced intensity conditioning compared with myeloablative conditioning using unrelated donor transplants in patients with acute myeloid leukemia," Journal of Clinical Oncology, vol. 27, no. 27, pp. 45704577, 2009.

[10] A. M. Raiola, A. Dominietto, A. Ghiso et al., "Unmanipulated haploidentical bone marrow transplantation and posttransplantation cyclophosphamide for hematologic malignancies after myeloablative conditioning," Biology of Blood and Marrow Transplantation, vol. 19, no. 1, pp. 117-122, 2013.

[11] H. J. Symons, A. Chen, C. Gamper et al., "Haploidentical BMT using fully myeloablative conditioning, $\mathrm{T}$ cell replete bone marrow grafts, and post-transplant cyclophosphamide (PT/Cy) has limited toxicity and promising efficacy in largest reported experience with high risk hematologic malignancies," Biology of Blood and Marrow Transplantation, vol. 21, no. 2, p. S29, 2015.

[12] D. Grosso, M. Carabasi, J. Filicko-O’Hara et al., “A 2-step approach to myeloablative haploidentical stem cell transplantation: a phase $1 / 2$ trial performed with optimized T-cell dosing," Blood, vol. 118, no. 17, pp. 4732-4739, 2011.

[13] D. Grosso, S. Gaballa, O. Alpdogan et al., "A two-step approach to myeloablative haploidentical transplantation: low nonrelapse mortality and high survival confirmed in patients with earlier stage disease," Biology of Blood and Marrow Transplantation, vol. 21, no. 4, pp. 646-652, 2015.

[14] S. R. McCurdy, J. A. Kanakry, M. M. Showel et al., "Riskstratified outcomes of nonmyeloablative HLA-haploidentical BMT with high-dose posttransplantation cyclophosphamide," Blood, vol. 125, no. 19, pp. 3024-3031, 2015.

[15] M. Aoudjhane, M. Labopin, N. C. Gorin et al., "Comparative outcome of reduced intensity and myeloablative conditioning regimen in HLA identical sibling allogeneic haematopoietic stem cell transplantation for patients older than 50 years of age with acute myeloblastic leukaemia: a retrospective survey from the Acute Leukemia Working Party (ALWP) of the European group for Blood and Marrow Transplantation (EBMT)," Leukemia, vol. 19, no. 12, pp. 2304-2312, 2005.

[16] M. Mohty, M. Labopin, L. Volin et al., "Reduced-intensity versus conventional myeloablative conditioning allogeneic stem cell transplantation for patients with acute lymphoblastic leukemia: a retrospective study from the European Group for Blood and Marrow Transplantation," Blood, vol. 116, no. 22, pp. 4439-4443, 2010.

[17] A. Shimoni, I. Hardan, N. Shem-Tov et al., "Allogeneic hematopoietic stem-cell transplantation in AML and MDS using myeloablative versus reduced-intensity conditioning: the role of dose intensity," Leukemia, vol. 20, no. 2, pp. 322-328, 2006.

[18] S. O. Ciurea, M.-J. Zhang, A. A. Bacigalupo et al., "Haploidentical transplant with posttransplant cyclophosphamide vs matched unrelated donor transplant for acute myeloid leukemia," Blood, vol. 126, no. 8, pp. 1033-1040, 2015.

[19] F. Ciceri, M. Labopin, F. Aversa et al., "A survey of fully haploidentical hematopoietic stem cell transplantation in adults with high-risk acute leukemia: a risk factor analysis of outcomes for patients in remission at transplantation," Blood, vol. 112, no. 9, pp. 3574-3581, 2008.

[20] S. O. Ciurea, V. Mulanovich, R. M. Saliba et al., "Improved early outcomes using a $\mathrm{T}$ cell replete graft compared with $\mathrm{T}$ cell depleted haploidentical hematopoietic stem cell transplantation," Biology of Blood and Marrow Transplantation, vol. 18, no. 12, pp. 1835-1844, 2012.

[21] B. Federmann, M. Bornhauser, C. Meisner et al., "Haploidentical allogeneic hematopoietic cell transplantation in adults using CD3/CD19 depletion and reduced intensity conditioning: a 
phase II study," Haematologica, vol. 97, no. 10, pp. 1523-1531, 2012.

[22] N. Cieri, G. Oliveira, R. Greco et al., "Generation of human memory stem T cells after haploidentical T-replete hematopoietic stem cell transplantation," Blood, vol. 125, no. 18, pp. 28652874, 2015.

[23] A. Roberto, L. Castagna, V. Zanon et al., "Role of naivederived $\mathrm{T}$ memory stem cells in T-cell reconstitution following allogeneic transplantation," Blood, vol. 125, no. 18, pp. 28552864, 2015.

[24] C. G. Kanakry, S. Ganguly, M. Zahurak et al., "Aldehyde dehydrogenase expression drives human regulatory $\mathrm{T}$ cell resistance to posttransplantation cyclophosphamide," Science Translational Medicine, vol. 5, no. 211, Article ID 211ra157, 2013.

[25] D. Ross, M. Jones, K. Komanduri, and R. B. Levy, "Antigen and lymphopenia-driven donor $\mathrm{T}$ cells are differentially diminished by post-transplantation administration of cyclophosphamide after hematopoietic cell transplantation," Biology of Blood and Marrow Transplantation, vol. 19, no. 10, pp. 1430-1438, 2013.

[26] P. V. O’Donnell, L. Luznik, R. J. Jones et al., "Nonmyeloablative bone marrow transplantation from partially HLA-mismatched related donors using posttransplantation cyclophosphamide," Biology of Blood and Marrow Transplantation, vol. 8, no. 7, pp. 377-386, 2002.

[27] A. Bashey, X. Zhang, K. Jackson et al., "Comparison of outcomes of hematopoietic cell transplants from T-replete haploidentical donors using post-transplantation cyclophosphamide with 10 of 10 HLA-A, -B, -C, -DRB1, and -DQB1 allele-matched unrelated donors and HLA-identical sibling donors: a multivariable analysis including disease risk index," Biology of Blood and Marrow Transplantation, vol. 22, no. 1, pp. 125-133, 2016.

[28] A. Bashey, X. Zhang, C. A. Sizemore et al., "T-cell-replete HLAhaploidentical hematopoietic transplantation for hematologic malignancies using post-transplantation cyclophosphamide results in outcomes equivalent to those of contemporaneous HLA-matched related and unrelated donor transplantation," Journal of Clinical Oncology, vol. 31, no. 10, pp. 1310-1316, 2013.

[29] A. Di Stasi, D. R. Milton, L. M. Poon et al., "Similar transplantation outcomes for acute myeloid leukemia and myelodysplastic syndrome patients with haploidentical versus 10/10 human leukocyte antigen-matched unrelated and related donors," Biology of Blood and Marrow Transplantation, vol. 20, no. 12, pp. 1975-1981, 2014. 


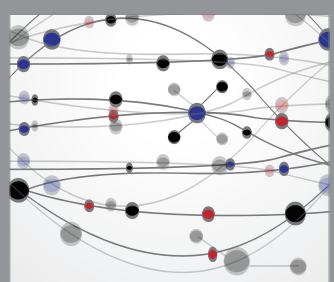

The Scientific World Journal
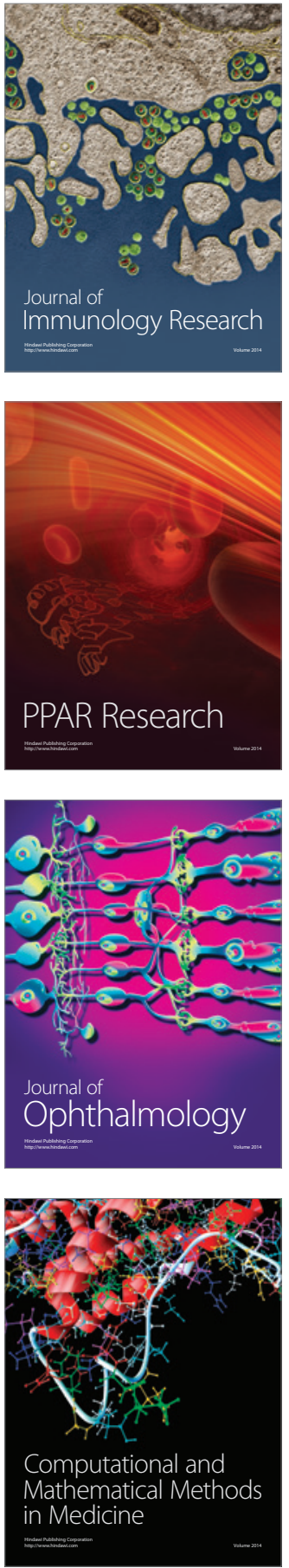

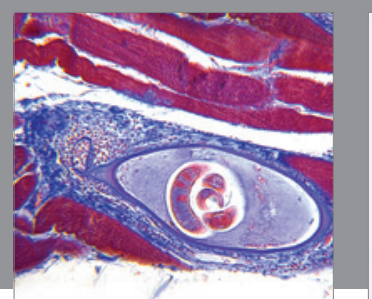

Gastroenterology Research and Practice

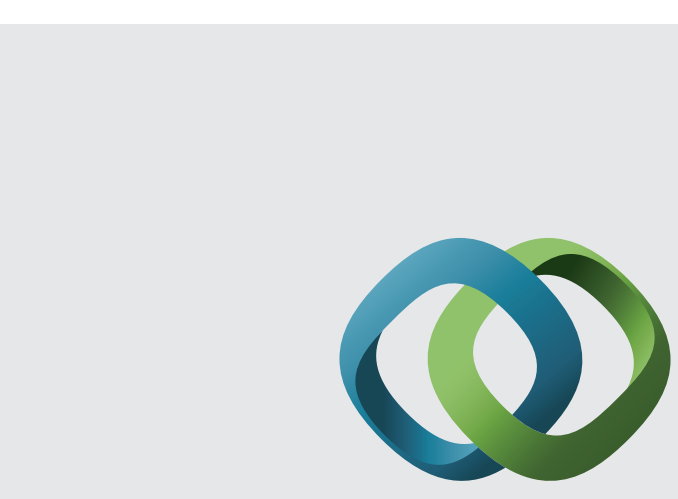

\section{Hindawi}

Submit your manuscripts at

http://www.hindawi.com
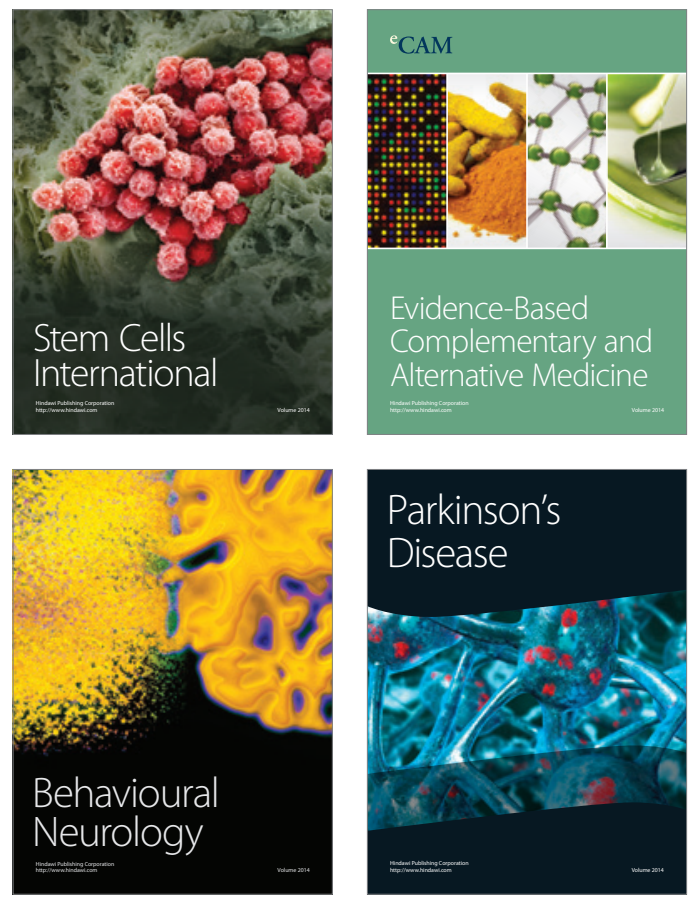
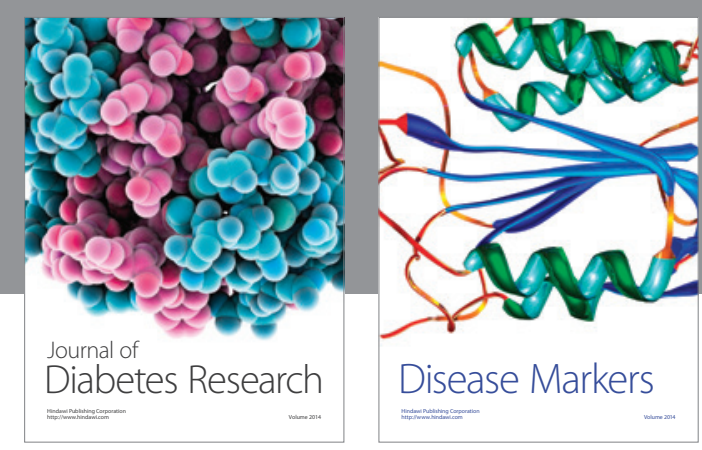

Disease Markers
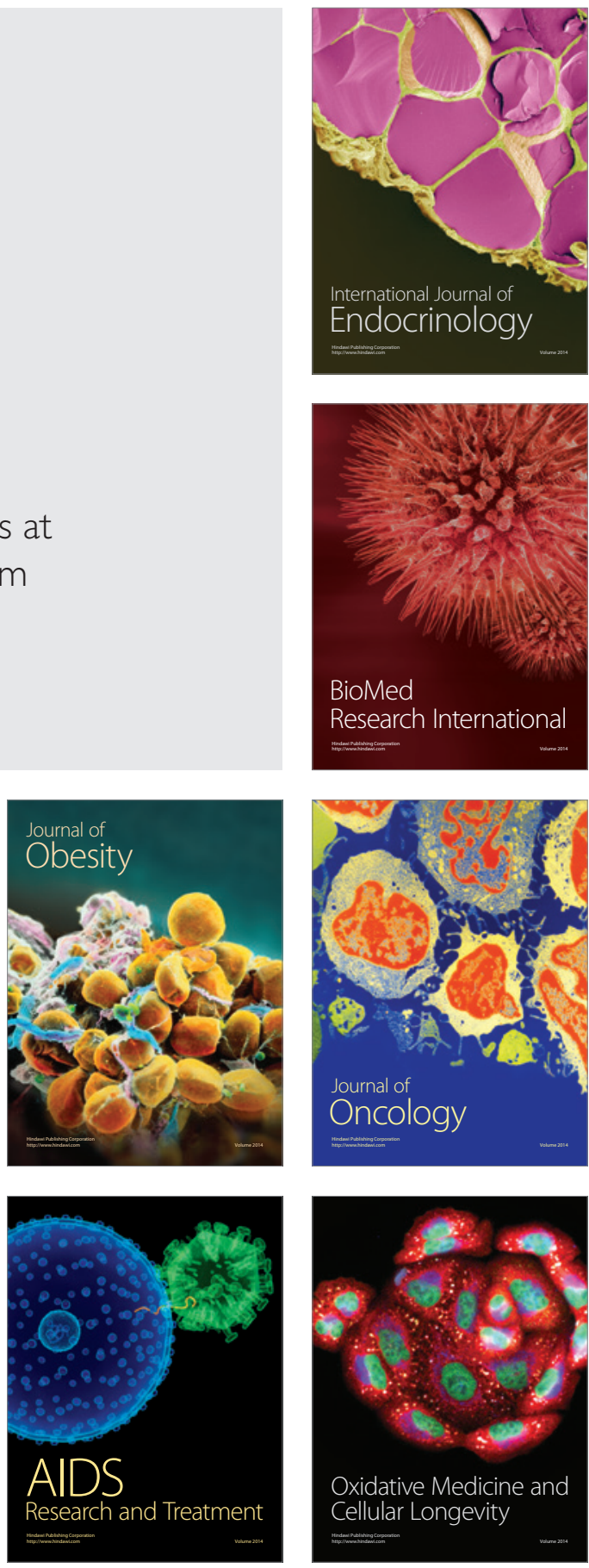